\title{
Influência do intervalo entre cortes sobre a produção de biomassa de duas espécies de capim limão
}

\author{
André May'; Odair A. Bovi'; Nilson B. Maia'; Andrea RA de Moraes²; Mariane Q Pinheiro²; Marcelo de \\ Mario $^{2}$ \\ ${ }^{1}$ Instituto Agronômico Campinas, C. Postal 28, 13012-970 Campinas-SP; ${ }^{2}$ Estagiário, Instituto Agronômico; amay@iac.sp.gov.br
}

\begin{abstract}
RESUMO
Foi estudada a influência do intervalo entre cortes na produção de massa seca da parte aérea de duas espécies de capim limão. O experimento foi realizado no Instituto Agronômico, em CampinasSP, 04 de junho/05 a 28 de agosto/06. O delineamento experimental utilizado foi em blocos ao acaso, com três repetições, com os tratamentos em esquema fatorial $2 \times 4$, sendo duas espécies (C. citratus e $C$. flexuosus) e quatro intervalos entre cortes $(40 ; 60 ; 80$ e 100 dias). Para $C$. flexuosus, maiores intervalos entre cortes proporcionaram maior massa seca acumulada ao longo do ciclo de cultivo, partindo de 329,04 para 704,16 g planta $^{-1}$ de massa seca acumulada da parte aérea, para intervalos entre cortes de 40 e 100 dias, respectivamente. A espécie $C$. citratus apresentou resposta linear decrescente da massa seca acumulada da parte aérea quanto maior o intervalo entre cortes utilizado, produzindo $238,68 \mathrm{~g}_{\text {planta }}{ }^{-1}$ utilizando intervalo entre cortes de 40 dias.
\end{abstract}

Palavras-chave: Cymbopogon citratus, Cymbopogon flexuosus, manejo cultural, planta medicinal, planta aromática.

\begin{abstract}
Influence of the interval between cuts on biomass yield of two lemon grass species

The aim of this study was to analyze the influence of the interval between cuts on the dry mass yield of the aerial part of two lemon grass species. The experiment was conducted at Agronomical Institute (IAC), in Campinas - SP, from June $4^{\text {th }}, 2005$ through August $28^{\text {th }}, 2006$. The experiment was carried out in a completely randomized block design, with three replications, and the treatments in a $2 \times 4$ factorial design, corresponding two species (C. citratus and $C$. flexuosus) and four intervals between cuts $(40 ; 60 ; 80$ and 100 days). For $C$. flexuosus, longer intervals between cuts generated more dry mass accumulated throughout the cultivation cycle, beginning on 329,04 to $704,16 \mathrm{~g} \mathrm{plant}^{-1}$ of accumulated dry mass of the aerial part, for intervals between cuts of 40 and 100 days, respectively. The $C$. citratus species showed a decrease in its linear response for the accumulated dry mass of the aerial part the longer the interval between cuts was, yielding 238,68 g plant $^{-1}$ for a 40 day interval between cuts.
\end{abstract}

Keywords: Cymbopogon citratus, Cymbopogon flexuosus, crop management, medicinal plant, aromatic plant.

\section{(Recebido para publicação em 10 de janeiro de 2007; aceito em 9 de julho de 2008)}

$\mathrm{O}$ capim limão é uma erva perene, ereta, cespitosa, de 0,6 até $3 \mathrm{~m}$ de altura, com caule rizomatoso muito ramificado, escuro, curto, semi-subterrâneo e palhoso. Suas raízes são fibrosas, escuras e numerosas. Dos rizomas partem colmos em tufos eretos e folhosos. Suas folhas verde-grisáceas com veios bem visíveis na face inferior e de cor verde-brilhante e lisa na face superior são moles, basais, planas, glabras, estreitas e longas ( $0,5 \mathrm{a} 1 \mathrm{~m})$; invaginantes, aromáticas, paralelinérveas, com margens ásperas e cortantes e ápice acuminado. As espiguetas situam-se sobre ráquis que formam racimos curtos (1 a 1,5 cm) que, por sua vez, formam panículas contraídas, bracteosas e terminais. O florescimento é muito raramente observado, na espécie Cymbopogon citratus. Os frutos são cariopses oblongas, secos indeiscentes (Lorenzi \& Matos, 2002; Castro \& Ra- mos, 2003). Já a espécie Cymbopogon flexuosus tem florescimento intenso durante os meses de inverno nas condições climáticas do Brasil.

O gênero Cymbopogon pertence à família Poaceae (Gramineae), sendo originário do Sul da Ásia, Sudeste Asiático e Austrália. Este gênero foi perfeitamente adaptado no Brasil, e é comumente encontrada nos estados de São Paulo; Minas Gerais; Pará; Pernambuco; Maranhão e da Bahia ao Rio de Janeiro.

É cultivada como ornamental e aromática e é utilizada como agente aromatizante em perfumaria e cosmética, devido ao seu odor característico de limão (Corrêa, 1984). Possui emprego medicinal, para o qual podem ser utilizadas as folhas frescas ou dessecadas e o óleo extraído delas onde se atribuem atividades sedativas, digestivas, anti-reumáticas, calmantes, antifebris, carminativas, estomáquicas, analgésicas, antiespasmódicas e antimicrobianas, além de ter propriedade de repelente de insetos (Corrêa Júnior et al., 1994).

O capim-limão apresenta duas espécies, com características diferentes. A primeira delas, de nome científico Cymbopogon flexuosus (D.C.) Stapf, é conhecida como capim-limão da Índia Oriental e o segundo, o Cymbopogon citratus (D.C.) Stapf é muito conhecido no Brasil como capim-limão, capimcidreira, erva-cidreira, capim-santo, cana-limão, capim-cidrão, capim-decheiro, capim-cidrilho, capim-cidró, capim-jossá, capim-marinho, chá-deestrada, erva-cidreira, citronela-de-java, capim-citronela, falso-patchuli, patchuli, verbena-da-Índia; e no exterior é denominado de capim limão da Guatemala (Lorenzi \& Matos, 2002).

No entanto, as informações sobre o 


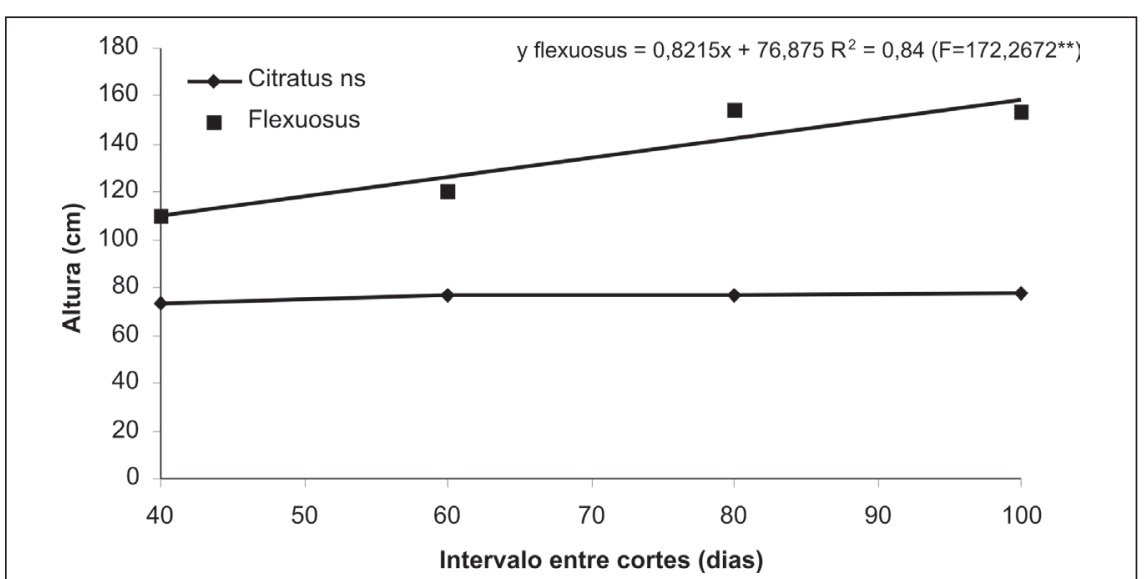

Figura 1. Influência do intervalo entre cortes sobre a altura da planta para cada espécie estudada. (Effects of interval between cuts on plant height, for each lemon grass species). Campinas, IAC, 2006.

cultivo e manejo cultural das duas espécies são escassas; portanto, neste trabalho visou-se estudar a influência do intervalo entre cortes de duas espécies de capim limão sobre a produção de biomassa.

\section{MATERIAL E MÉTODOS}

O experimento foi realizado no Centro Experimental do Instituto Agronômico (IAC), em Campinas-SP, de 04/07/05 a 28/08/06. O delineamento experimental utilizado foi em blocos ao acaso, com três repetições, com os tratamentos em esquema fatorial $2 \times 4$, sendo duas espécies (C. citratus e $C$. flexuosus) e quatro intervalos entre cortes $(40 ; 60 ; 80 \mathrm{e}$ 100 dias).

O material genético (perfilhos) utilizado pertencia ao banco de germoplasma do IAC - Coleção de Plantas Aromáticas e Medicinais, provenientes de plantas com dois anos idade, cuja exsicata presente no Herbário do IAC é mantida pelo número 45335 . Os perfilhos foram transferidos para sacos plásticos contendo terra de barranco misturada com esterco de curral curtido, para enraizamento, sendo transplantados para o campo decorrido 45 dias.

O solo do local da experimentação é classificado como um LATOSSOLO VERMELHO AMARELO, textura argilosa (EMBRAPA, 1999). A análise do solo na profundidade de 0 a $0,2 \mathrm{~m}$ revelou: $\mathrm{pH}$ em $\mathrm{CaCl}_{2}$ de 6,0 e $34 \mathrm{~g} \mathrm{dm}^{-3}$ de matéria orgânica, além de $56 \mathrm{mg} \mathrm{dm}^{-3} \mathrm{de}$ P-resina. Os teores de $\mathrm{K}, \mathrm{Ca}, \mathrm{Mg}, \mathrm{H}+\mathrm{Al}$ foram, respectivamente, 2,$2 ; 64 ; 21$ e 22 $\mathrm{mmol}_{\mathrm{c}} \mathrm{dm}^{-3} \mathrm{e}$ a CTC efetiva igual a 109,7 $\mathrm{mmol}_{\mathrm{c} .} \mathrm{dm}^{-3}$, segundo metodologia descrita por Raij et al. (2001).

O campo experimental foi preparado com adubação orgânica, na dose de $5 \mathrm{~kg} \mathrm{~m}^{-1}$ de esterco curtido de bovinos (Corrêa Júnior et al., 1994). O espaçamento utilizado foi de $0,8 \mathrm{~m}$ entre linhas por 0,5 m entre plantas. Cada parcela foi composta por 15 plantas, sendo avaliadas as três plantas centrais.

As irrigações, por aspersão convencional, foram diárias durante as primeiras semanas após o transplantio das mudas para o campo, até o pegamento completo das mudas, e semanalmente em complementação às precipitações pluviais, nos períodos subseqüentes, com a aplicação de uma lâmina média de $10 \mathrm{~mm}$.

Foram feitas avaliações, segundo o intervalo de corte estudado, das características: altura da planta e massa seca da parte aérea acumulada.

Para as condições do experimento no momento da definição da altura de corte para cada espécie estudada, foram estipuladas as alturas de corte de $40 \mathrm{~cm}$ para a espécie $C$. citratus e de $60 \mathrm{~cm}$ para $C$. flexuosus, em virtude do vigor pessoal, Nappi S.A., Sítio ${ }^{\text {ta }}$ Rita, São José do Rio Pardo-SP).

Foi feito corte de uniformização em todas as plantas do experimento em 11/ $11 / 05$, visando iniciar a contagem do intervalo de corte de cada tratamento estudado. Dessa forma foram realizados diferencial de cada espécie (Informação
6; 3; 3 e 2 cortes respectivamente para os intervalo entre cortes de 40;60; 80 e 100 dias. A altura da planta foi aferida com o auxílio de fita métrica, medindose a distância da superfície do solo até a parte superior de cada planta amostrada.

Para a secagem da parte aérea coletada ao longo do período experimental segundo cada intervalo de corte estudado, foi utilizada uma estufa com circulação forçada de ar quente a $65^{\circ} \mathrm{C}$, pelo período de 96 horas até atingir massa constante.

O estudo das características estudadas foi feito graficamente através de regressões polinomiais pelo programa estatístico ESTAT (UNESP-FCAV, Campus Jaboticabal). Os modelos de regressão foram escolhidos pelo maior coeficiente de determinação $\left(\mathrm{R}^{2}\right)$ que tenha apresentado maior significância pelo teste $\mathrm{F}$.

\section{RESULTADOS E DISCUSSÃO}

A espécie $C$. flexuosus apresentou incrementos lineares da altura da planta quanto maior o intervalo entre cortes utilizado (Figura 1), apresentando valores de 110 a $159 \mathrm{~cm}$ para intervalo entre cortes de 40 e 100 dias, respectivamente. Assim, quando mais intensa é a freqüência de corte (intervalos entre cortes menores), menor o crescimento da planta em altura, em razão do menor período de rebrota imposto.

Não houve ajuste dos valores médios observados para a característica altura da planta para espécie $C$. citratus para nenhum dos modelos de regressão polinomial utilizado. As médias das alturas das plantas dessa espécie apresentaram valores muito próximos entre si, em torno de $76 \mathrm{~cm}$, para qualquer intervalo entre corte estudado.

No entanto, para $C$. flexuosus, maiores intervalos entre cortes proporcionaram maior massa seca acumulada ao longo do ciclo de cultivo. Verificaram-se incrementos lineares na massa seca acumulada da parte aérea quanto maior o intervalo entre cortes utilizado, partindo de 329,04 g planta $^{-1}$ para 704,16 g plan$\mathrm{ta}^{-1}$ de massa seca acumulada da parte aérea, para intervalos entre cortes de 40 e 100 dias, respectivamente (Figura 2).

O C. citratus, por outro lado, apre- 
sentou uma resposta oposta, havendo um decréscimo linear da massa seca acumulada da parte aérea quanto maior o intervalo entre cortes utilizado. Dessa forma, maiores produções de massa seca ao longo do ciclo foram obtidas com intervalos menores entre cortes $(238,68$ g planta $^{-1}$ ) (Figura 2). No entanto, considerando o intervalo entre cortes de 40 dias, para a obtenção da máxima produção de massa seca, a espécie $C$ citratus foi $24 \%$ menos produtiva comparativamente à menor produção de massa alcançada pelo C. flexuosus, no mesmo intervalo entre cortes considerado, demonstrando uma capacidade produtiva dessa última espécie muito superior.

São escassas as informações disponíveis sobre a influência do intervalo entre cortes sobre o gênero Cymbopogon. Alguns resultados indicam que a colheita do capim-limão deva ser feita a cada 120 dias para a espécie C. citratus e para C. flexuosus antes do florescimento (IAC, 1987).

Segundo Corrêa Júnior (1994) o primeiro corte da parte aérea da espécie $C$. citratus deve ser feito apenas após o sexto mês de cultivo, sendo possível 2 cortes por ano, atingindo uma produção de 2 a 3 t ha $^{-1}$ massa seca da parte aérea em um ano de cultivo, utilizando o espaçamento 1 x $0,5 \mathrm{~m}$, o que conferiu uma produção de 100 a 150 g planta $^{-1}$.

Blank et al. (2003), estudando o efeito do espaçamento, doses de biofertilizante e colheitas na produção de biomassa de capim-limão, verificaram maiores rendimentos de biomassa seca nas oito primeiras colheitas $(1,5 \mathrm{t}$ $\mathrm{ha}^{-1}$ de massa seca), utilizando o espaçamento $0,3 \times 0,3 \mathrm{~m}$, o que conferiu uma produção de $13,5 \mathrm{~g} \mathrm{planta}^{-1}$.

Salerno et al. (2004) citam que em lavouras comerciais é possível fazer três a quatro cortes por ano, produzindo $18 \mathrm{t}$ $\mathrm{ha}^{-1}$ de folhas frescas por ano, utilizando o espaçamento de $1 \mathrm{~m}$ entre linhas $\mathrm{x}$ 0,5 m entre plantas, representando uma produção de $900 \mathrm{~g} \mathrm{planta}^{-1}$. Segundo Leal et al. (2003), em plantas de $C$. citratus, 29,4\% da massa fresca total da parte aérea corresponde à massa seca, portanto a produção obtida por Salerno et al. (2004) corresponderia a 5,3 $\mathrm{t} \mathrm{ha}^{-1}$ de massa seca de folhas, representando

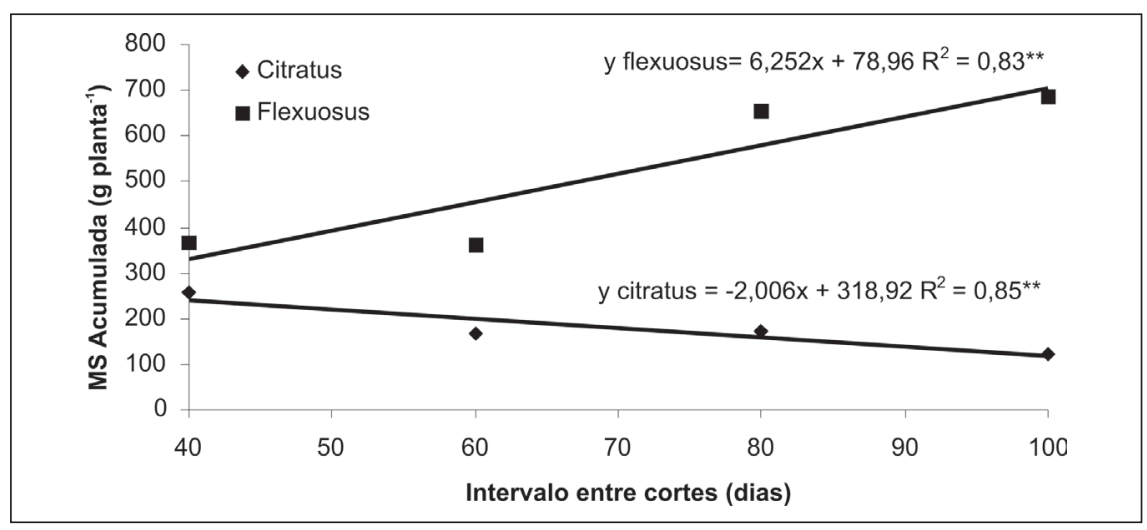

Figura 2. Influência do intervalo entre cortes sobre a massa seca da parte aérea acumulada (MS Acumulada) para cada espécie estudada. (Effects of interval between cuts on accumulated dry mass of aerial part (accumulated DM) for each lemon grass species). Campinas, IAC, 2006.

uma produção de $265 \mathrm{~g}_{\text {planta }}{ }^{-1}$.

A produção de biomassa por planta por corte variou conforme o intervalo entre cortes utilizado para cada espécie estudada, de forma que, maiores massas secas da parte aérea foram alcançadas por planta em maiores intervalos entre cortes, demonstrando que a planta, principalmente a espécie $C$. flexuosus, necessitou de maior período de recuperação, resultando em maiores produções. Na Figura 2, pode-se verificar uma elevação na massa seca da parte aérea com intervalos entre cortes maiores, apresentando os seguintes valores médios por corte para a espécie $C$. flexuosus: 54,84; 151,36; 193,04; 352,08 g planta ${ }^{-1}$ corte $^{-1}$, respectivamente, para os intervalos entre cortes 40;60; $80 \mathrm{e}$ 100 dias. Já a espécie $C$. citratus, mesmo com maiores períodos de recuperação da planta (maiores intervalos entre cortes), não teve o mesmo comportamento, pois a produção de biomassa por planta por corte para cada intervalo entre cortes manteve valores próximos entre si $(39,78 ; 66,19 ; 52,81$ e 59,16 g planta $^{-1}$ corte $^{-1}$, respectivamente, $40 ; 60$; 80 e 100 dias).

Mesmo nos intervalos entre cortes menores não houve mortalidade de plantas, demonstrando ainda capacidade produtiva das plantas além do período estudado.

A espécie C. flexuosus é caracterizada pelo florescimento intenso durante os meses de baixas temperaturas, sendo que nas condições do experimento ocorreu a partir de 10/07/06. O cresci- mento da parte aérea dessa espécie após o início do florescimento foi bastante reduzido, principalmente após 240 dias do corte de uniformização.

A espécie $C$ citratus apresentou sintomas do ataque de Curvularia andropogonis (Monteiro \& Barreto, 2002) durante os meses de inverno quando as plantas reduziram o crescimento da parte aérea. A espécie $C$. flexuosus não apresentou sintomas da doença nas condições do experimento. $\mathrm{O}$ fungo foi identificado pelo Centro de Fitopatologia do Instituto Agronômico quando do início da sua ocorrência nas plantas, visando à confirmação da doença.

Os resultados permitem concluir que a espécie $C$. flexuosus foi mais produtiva que a $C$. citratus; a espécie $C$. flexuosus apresentou maior produção de massa seca para maiores intervalos entre cortes, sendo observado resposta contrária para a espécie $C$. citratus.

\section{REFERÊNCIAS}

BLANK AF; SANTANA FILHO L GM; AMÂNCIO VF; SILVA PA; CARVALHO FILHO JLS; OLIVEIRA AS; SANTOS MF; ANDRADE LG; DANTAS IB; COSTA AG; AZEVEDO VG; MENDONÇA MC; ARRIGONI-BLANK MF; SILVA-MANN R. 2003. Influência de espaçamento, doses de biofertilizante e colheitas na produção de biomassa de capim-limão (Cymbopogon citratus (D.C.) Stapf - Poaceae). In: CONGRESSO BRASILEIRO DE OLERICULTURA, 43, Resumos...Recife: SOB (CD-ROM).

CASTRO LO; RAMOS RLD. 2003. Principais gramíneas produtoras de óleos essenciais. Boletim Técnico da Fundação Estadual de 
Pesquisa Agropecuária (FEPAGRO). Secretaria da Ciência e Tecnologia. Rio Grande do Sul. n.11, 28 p.

CORRÊA MP. 1984. Dicionário das plantas úteis do Brasil e das exóticas cultivadas. Rio de Janeiro, Nacional, v.1, p. 577.

CORRÊA JÚNIOR C; MING CL; SCHEFFER MC. 1994. Cultivo de plantas medicinais, condimentares e aromáticas. Jaboticabal/SP: FUNEP, 162p.

IAC. 1987. Instruções agrícolas para o Estado de São Paulo. 4 ed. Campinas/SP, 231p.
EMBRAPA. 1999. Sistema Brasileiro de Classificação de Solos. Rio de Janeiro: Embrapa Solos, 412p.

LEAL TCAB; FREITAS SP; SILVA JF; CARVAlHO AJC. 2003. Produção de biomassa e óleo essencial em plantas de capim cidreira [Cymbopogon citratus (Dc.) Stapf.] em diferentes idades. Revista Brasileira de plantas Medicinais 5: 61-4.

LORENZI H; MATOS FJA. 2002. Plantas medicinais no Brasil: nativas e exóticas. Instituto Plantarum de estudos e da flora, p. 252.

MONTEIRO FT; BARRETO RW. 2002. Curvularia andropogonis: agente etiológico da queima foliar do capim-limão. Fitopatologia Brasileira 27: 227.

RAIJ B; ANDRADE JC; CANTARELLA H; QUAGGIO JÁ. 2001. Análise química para avaliação da fertilidade de solos tropicais. Campinas: Instituto Agronômico, 285p.

SALERNO AR; AGOSTINI I; SILVA JÚNIOR AA. 2004. Normas técnicas para cultivo de capim-limão, citronela, palma-rosa e patchuli. Florianópolis: EPAGRI, 58p (Sistema de Produção, 37). 